\title{
Avaliação ambiental do rio Pandeiros utilizando macroinvertebrados como indicadores de qualidade da água
}

\author{
Renan de Souza Rezende ${ }^{13, \otimes}$; Anderson Medeiros Dos Santos ${ }^{1} \&$ José F. Gonçalves Junior ${ }^{2}$ \\ 1. Universidade Estadual de Montes Claros, Brasil. 2. Universidade de Brasília, Brasília, Brasil. 3. Universidade Federal de \\ Santa Catarina. Campus Universitário Trindade, Laboratório de Limnologia, Florianópolis, Brasil.
}

\begin{abstract}
Resumo. O objetivo deste trabalho foi avaliar as condições ecológicas da bacia do rio Pandeiros com base em métricas das comunidades de invertebrados bentônicos (coletados por meio de Surber), amostradas em 20 pontos de fevereiro, maio, setembro e novembro de 2008 ao longo de uma bacia e as respectivas características limnológicas de cada local. Para isso foram calculadas, levando em consideração como replicas os quatro períodos amostrais (fevereiro, maio, setembro e novembro de 2008), a porcentagem de Ephemeroptera, Plecoptera e Trichoptera (\%EPT), o índice de qualidade de água BMWP-CETEC (Biological Monitoring Working Party) e o Protocolo de avaliação rápida. Um total de 17468 invertebrados foram coletados, distribuídos em 67 taxa. A Classe Insecta apresentou maior número de indivíduos e se distribuiu em 52 famílias. A elevada diversidade de taxa indica uma alta preservação da bacia com potencialidade em atuar como área de referência para avaliar a qualidade de água em outras bacias hidrográficas. Os trechos alto e médio da bacia apresentaram boa qualidade da água e não distinguiu entre si. O trecho mais a jusante da bacia, principalmente perto da foz com o Rio São Francisco, é a região mais urbanizada e com grande atividade agropecuária, apresentando os piores índices de qualidade da água. Ambas as métricas utilizadas baseadas na comunidade de invertebrados bentônicos (\%EPT e BMWP-CETEC) e o Protocolo de Avaliação Rápida foram eficazes na avaliação de impactos antrópicos. Assim estes resultados apesar de serem específicos para bacia do rio Pandeiros permitem que suas conclusões gerais possam ser utilizadas para outros locais de região tropical do bioma Cerrado.
\end{abstract}

[Palabras clave: ecologia de rios, EPT, BMWP-CETEC, protocolo de avaliação rápida]

\begin{abstract}
AвSTRACT. Environmental assessment of the Pandeiros river using macroinvertebrates as indicators of water quality: The aim of this study was to assess the ecological conditions of the Pandeiros River Watershed using the metrics of benthic macroinvertebrate communities (collected by Surber), sampling at 20 points (including their limnological characteristics) among February, May, September and November 2008 along watershed. We calculated, taking into account the replicas as four sampling periods (February, May, September and November 2008), the percentage of Ephemeroptera, Plecoptera and Trichoptera (\% EPT), the index of water quality-CETEC BMWP (Biological Monitoring Working Party) and the Protocol of rapid assessment. A total of 17468 invertebrates were collected, distributed in 67 taxa. The Insecta class had the greatest number of individuals and was distributed in 52 families. The high diversity indicates a higher rate of preservation of the watershed with the potential to act as a reference for assessing the quality of water in other watersheds. The middle and upper stretches of the water had good water quality and did not distinguish between them. The section further down the watershed, particularly near the mouth of the Rio Sao Francisco, is the most urbanized region, with large agricultural and livestock activity, with the worst indices of water quality. Both community-based metrics used for benthic invertebrates (\% EPT and BMWP-IEF) and the Rapid Assessment Protocol were effective in the evaluation of human impacts. So although these results are specific to Pandeiros River Watershed allow its general conclusions could be used for other sites of the tropical zone.
\end{abstract}

[Keywords: stream ecology, EPT, BMWP, watershed]

\section{INTRODUÇ̃̃O}

Os impactos ambientais nos ecossistemas aquáticos são um tema atual que ganha notoriedade, visto a importância dos recursos hídricos para processos industriais, agrícolas e urbanos (Dudgeon et al. 2006). Uma baixa qualidade de água pode ser decorrente de várias atividades antrópicas como: a mineração, assoreamento dos rios, lançamento de efluentes sem tratamento, utilização de agrotóxicos, retirada da vegetação ripária,

$\bowtie$ Universidade Federal de Santa Catarina. Campus Universitário Trindade, Laboratório de Limnologia. Florianópolis, SC. CEP 88040-970, Brasil. renanrezende30@gmail.com. construção de represas, retificação e desvio do curso natural de rios, esgotamento de recursos pesqueiros e introdução de espécies exóticas (Allan 2004; Scrimgeour \& Kendall 2003). A conservação dos ecossistemas aquáticos assegura os chamados "serviços ambientais" ou "serviços do ecossistema" (fornecimento de água para abastecimento doméstico e industrial, geração de energia elétrica, lazer, irrigação de culturas e conservação da vida silvestre) (Dudgeon et al. 2006).

Editor asociado: Fernando Unrein

Recibido: 10 de febrero de 2012; Fin de arbitraje: 11 de abril; Revisión recibida: 2 de mayo; Aceptado: 29 de mayo. 
Assim, fazem-se necessárias abordagens em escalas maiores quando comparados às escalas locais (e.g., trecho de rio) nos estudos ecológicos, chamando a atenção para trabalhos que utilizam a bacia hidrográfica como unidade de estudo (Beeby 1993; Cortes et al. 2011). A bacia hidrográfica é uma das unidades elementares da paisagem terrestre que possui importância para o desenvolvimento de práticas conservacionistas (Magnusson 2001). Além disso, é facilmente reconhecida como a área delimitada pelo relevo que drena a água da chuva por uma rede de córregos e rios, integrando o sistema terrestre e aquático local e regionalmente, através do fluxo de materiais (Nessimian et al. 2008). Utilizando esta abordagem, o biomonitoramento realizado na Bacia Hidrográfica do Rio das Velhas no Estado de Minas Gerais, é um exemplo raro e bem sucedido no Brasil (Callisto \& Moreno 2008; Paz et al. 2008). Desta forma, existe a necessidade de que este tipo de estudo seja empregado em outras áreas, como a Bacia Hidrográfica do Rio Pandeiros, um importante tributário da margem esquerda do Rio São Francisco que abriga o único pântano do Estado de Minas Gerais, e que não apresenta trabalhos desta natureza.

Dentre as formas de avaliação, controle, manejo e conservação destes ecossistemas, os programas de biomonitoramento têm obtido respostas precisas e seguras (Callisto \& Gonçalves 2005; Moreno \& Callisto 2005; Gualdoni et al. 2011). O biomonitoramento pode ser definido como sendo o "uso sistemático de respostas biológicas para avaliar mudanças no ambiente com o objetivo de utilizar estas informações em programas de controle de qualidade ambiental" (Rosenberg \& Resh 1993). Estes programas conjugam parâmetros abióticos e bióticos, os quais são identificadas espécies e/ou comunidades sensíveis aos impactos ocorridos nos ecossistemas (Stark et al. 2001). Estas comunidades, grupos de organismos e/ou espécies identificadas são classificados como bioindicadores, cuja presença, quantidade e distribuição indicam a magnitude de impactos ambientais em um ecossistema aquático e sua bacia de drenagem (Rosenberg \& Resh 1993; Dolédec et al. 2011). A utilização desta abordagem permite a avaliação integrada das fontes de poluição e torna-se mais eficiente do que as medidas diretas de parâmetros físicos e químicos (e.g., temperatura, $\mathrm{pH}$, oxigênio dissolvido, teores totais e dissolvidos de nutrientes, etc.) que são normalmente medidos em campo e/ou laboratório e utilizados para avaliar a qualidade das águas (Dolédec et al. 2011; Yung-Chul et al. 2011).

Dentre os bioindicadores, que podem ser peixes, plâncton, macrófitas aquáticas, microrganismos, dentre outros. Os macroinvertebrados bentônicos têm sido utilizados frequentemente na avaliação de impactos ambientais e saúde dos ecossistemas (Rosenberg \& Resh 1993; Metzeling et al. 2003). Macroinvertebrados bentônicos são organismos com tamanho de corpo maior que $0.5 \mathrm{~mm}$, que habitam o sedimento de ecossistemas aquáticos continentais (e.g., moluscos, anelídeos, insetos) (Morgan et al. 2006). Estes organismos colonizam substratos como restos de troncos, acúmulos de folhas, pedras, macrófitas aquáticas, algas filamentosas, durante parte ou por todo o seu ciclo de vida (Morgan et al. 2006). O biomonitoramento pela comunidade de macroinvertebrados bentônicos possui diversas vantagens, dentre estas estão à rapidez e eficiência na obtenção de resultados, baixo custo, maior susceptibilidade a uma grande variedade de estressores, assim como avaliação e monitoramento ambiental de um ecossistema em nível de bacia hidrográfica (Rosenberg \& Resh 1993). Além destas características o uso dos bioindicadores permite o desenvolvimento de índices e métricas de integridade biótica, que facilitam e padronizam a avaliação ambiental em diferentes locais (Ferreira et al. 2011).

Em 1970 na Inglaterra se tem o primeiro registro oficial do uso de um método biológico para avaliar a qualidade da água de rios (Hawks 1997; Ferreira et al. 2011). Posteriormente, foi desenvolvido em 1976 o primeiro índice BMWP (Biological Monitoring Working Party), que é muito recomendado para avaliação da condição ambiental dos rios. Atualmente, estes índices são muito variáveis e sofreram diversas adequações para a realidade da unidade de estudo e por isso são amplamente aplicados nos últimos 15 anos, ao longo de todo o mundo (Hawks 1997; Smith et al. 1999; Stoddard et al. 2005; Ferreira et al. 2011).

Segundo, Junqueira \& Campos (1998), apesar do grande número de índices desenvolvidos, a maior parte é destinada as regiões temperadas, que apresenta uma biota mais conhecida e taxonomicamente definida quando comparado às regiões tropicais. Este fato ocorre devido a enorme diversidade biológica e sua consequente falta de detalhamento taxonômico para diversos grupos tropicais, 
impedindo a utilização eficiente destes índices. Assim, no Brasil métricas como a porcentagem de Ephemeroptera, Plecoptera e Trichoptera (\%EPT) e o índice BMWPCETEC merecem destaque, pois apresentam uma exigência taxonômica baixa (ordem e família, respectivamente) para sua aplicação, possuem também baixo custo, são amplamente utilizadas, possuem diversas adaptações para os sistemas tropicais e são diretamente correlacionadas a qualidade ambiental (Junqueira \& Campos 1998; Ferreira et al. 2011), permitindo e viabilizando o monitoramente por longos períodos. Os organismos pertencentes às ordens Ephemeroptera, Plecoptera e Trichoptera, são considerados excelentes indicadores da condição ambiental em todos os ambientes, pois estes são extremamente sensíveis à poluição, constituindo uma ferramenta útil na avaliação do meio (Bouchard 2004). O índice $\mathrm{BMWP}$, apresenta algumas adaptações para sub-bacias brasileira, como o BMWP-CETEC que foi elaborado para uma das bacias (rio das Velhas) do rio São Francisco (Junqueira \& Campos 1998).

Assim, as métricas construídas a partir da comunidade de macroinvertebrados bentônicos são uma forma importante para caracterizar a qualidade dos corpos aquáticos tornando o monitoramento ambiental economicamente viável e de fácil demonstração para os tomadores de decisão (Sandin \& Johnson 2000). Para avaliação e monitoramento ambiental de uma região, através de bioindicadores em corpos aquáticos, é necessário conhecer o funcionamento do sistema, assim como suas comunidades originais, antes que estes sejam impactados, definindo como áreas ou pontos de referência para estudos de qualidade de água, quando comparados com outros pontos e bacias adjacentes (Gualdoni et al. 2011). Outro aspecto importante é que o monitoramento ambiental permite verificar se políticas conservacionistas como a implantação de Áreas de Proteção Ambiental (APA) são eficazes para a proteção da biodiversidade e manutenção dos serviços ecológicos, justificando a importância deste trabalho na APA da bacia do rio Pandeiros. Permite também a detecção de áreas com maior fragilidade ambiental, possibilitando concentrar esforça em sua conservação. A partir de métricas biológicas é possível então que este monitoramento ocorra de forma rápida, eficiente e com baixo custo reduzindo a necessidade de analises abióticas custosa para verificar a qualidade ambiental na APA da bacia do rio Pandeiros ou outras adjacentes (Gualdoni et al. 2011).

Neste contexto o presente estudo teve como objetivo avaliar as condições ecológicas na APA da bacia do rio Pandeiros, com base em duas métricas obtidas a partir da comunidade de macroinvertebrados aquáticos (BMWP-CETEC e \%EPT) e no "Protocolo de Avaliação Rápida das Condições Ecológicas e da Diversidade de Habitats em Trechos de Bacias Hidrográficas" (Callisto et al. 2002).

\section{MÉTOdos}

\section{Área de estudo}

O estudo foi realizado na APA da bacia do Rio Pandeiros, localizada no estado de Minas Gerais, sudeste do Brasil, que abrange os municípios de Januária, Bonito de Minas e Cônego Marinho. Esta possui uma área de 380000 ha. Esta é caracterizada pela a sua baixa densidade demográfica (8164 habitantes), vivendo em pequenas comunidades rurais, subsistindo da agricultura e a pecuária de pequena escala (Fonseca et al. 2008). Esta região engloba áreas de transição entre os biomas Cerrado e Caatinga, e possui o único pântano do estado de Minas Gerais. A vegetação predominante é o cerrado, ocupando cerca de $48 \%$ da área. Floresta riparia e veredas somam $20 \%$ da bacia do rio Pandeiros e a agricultura e pecuária, em sua maioria de subsistência, encontra-se distribuídas ao longo da bacia, com área de 31\%. Áreas urbanas apresentam baixos percentuais (1\%). O clima predominante é o Semiárido, temperatura variando entre 18 e $35^{\circ} \mathrm{C}$. A altitude média da região varia de 600 a 640 m e o solo predominante é o Latossolo vermelho com textura arenosa e areias quartizosas (Fonseca et al. 2008). O Rio Pandeiros possui aproximadamente $145 \mathrm{~km}$ de extensão, e seus principais afluentes pela margem esquerda são os rios Pindaibal, São Pedro, Alegre e Mandins (todos de terceira ordem), enquanto os da margem direita são os rios São Domingos (quarta ordem), Catolé (quarta ordem), Borrachudo (terceira ordem) e Macaúbas (terceira ordem) (Strahler 1963) (Figura 1; Tabela 1 do Material Suplementar).

\section{Comunidade de macroinvertebrados bentônicos}

As coletas ocorreram em 20 locais ao longo da bacia do rio Pandeiros. Destes, oito pontos foram no Rio Pandeiros, nove pontos em seus afluentes e três pontos na região de pântano, nos meses de fevereiro, maio, setembro e novembro de 2008. Dos pontos coletados, seis pontos foram na parte superior da bacia, oito pontos na parte média e seis pontos na porção próxima a foz da Bacia do Rio Pandeiros (Figura 1). Esta é uma divisão que leva em conta a localização geográfica do ponto de coleta da bacia hidrografia como proposto através da base hidrográfica Otto-codificada da Agencia 


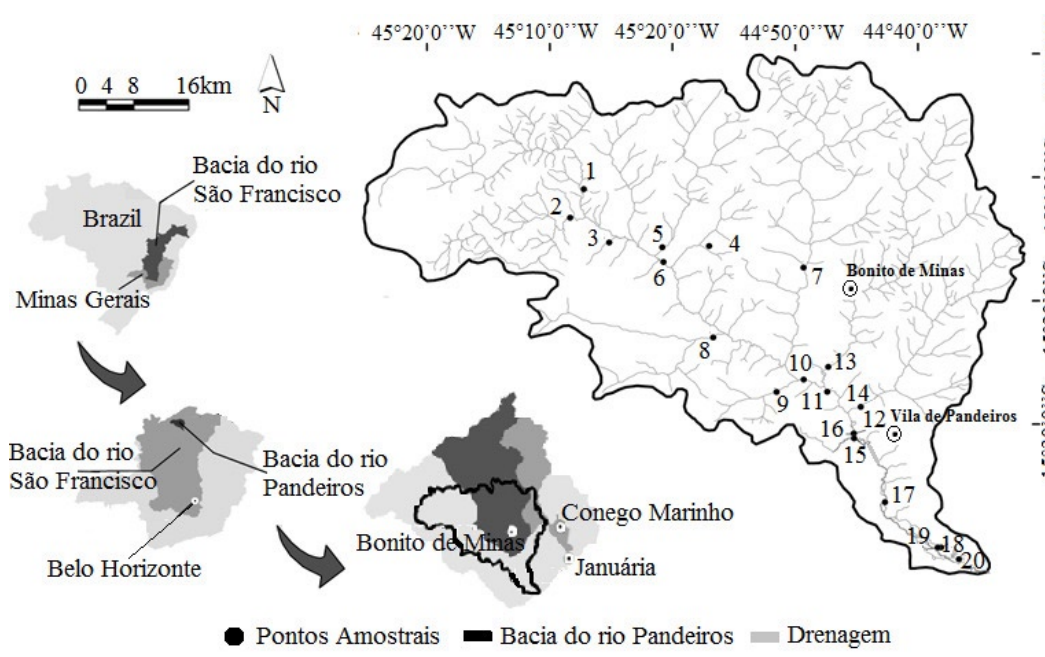

2
$\vdots$
0
$\vdots$
$\cdots$

Figura 1. Mapa com a - localização da bacia do rio Pandeiros em relação ao país, estado de Minas Gerais, bacia do rio São Francisco, cidades pertencentes e a distribuição dos pontos ao longo da in mesma. Localização da Vila de Pandeiros.

Figure 1. Map showing the location of the Pandeiros river basin for the country, of Minas Gerais state, São Francisco river basin, city belonging and the distribution of points along the same. Location of the

Nacional de Águas (ANA) do Brasil (Tabela 1 do Material Suplementar). Para estimar a comunidade bentônica três amostras de sedimento foram coletadas, em cada estação amostral, por meio de um coletor do tipo "Surber", com área amostral de $1024 \mathrm{~cm}^{2}$ e malha de $0.250 \mathrm{~mm}$ (Merrit \& Cummins 1996; Roldán 1988). As amostras foram lavadas sobre peneiras de $0.50 \mathrm{~mm}$, triadas com auxílio de estereomicroscópio e os invertebrados aquáticos identificados até o nível família segundo bibliografia adequada (Merritt \& Cummins 1996; Roldán 1988).

Com base no inventário das comunidades de macroinvertebrados bentônicos foi calculado a porcentagem de EPT e o BMWP-CETEC/1998, O BMWP-CETEC é uma métrica usada para avaliação rápida da integridade biótica da água que leva em consideração a riqueza específica, atribuindo uma pontuação distinta para cada táxon. Os resultados obtidos por esta métrica divide a qualidade de água em cinco classes, que são ótima ( $\geq 86)$, boa (65-85), satisfatória (37-63), ruim (17-36) e péssima ( $\leq 16)$. Para maiores esclarecimentos sobre a pontuação atribuída a cada taxa consulte Junqueira \& Campos (1998). Para o calculo da porcentagem de EPT utilizou os valores médios dos quatro meses de coleta, e o calculo do BMWP-CETEC, foi feito levando em consideração a riqueza acumulada dos quatro meses de coleta.

\section{Variáveis físicas e químicas do trecho de rio}

Utilizou-se um "Protocolo de Avaliação Rápida das Condições Ecológicas e da Diversidade de Habitats em Trechos de Bacias Hidrográficas" (Protocolo de Avaliação Rápida), que busca avaliar não só o ambiente aquático, mas também o uso e ocupação do solo nas margens do rio (Callisto et al. 2002), e que foi criado para avaliar rios do bioma Cerrado, das sub-bacias do rio São Francisco, local onde pertence o rio Pandeiros, justificando sua utilização para este trabalho. Ele avalia um conjunto de parâmetros em categorias descritas e pontuadas de 0 a 5 ou de 0 a 4 . O valor final do protocolo de avaliação é obtido a partir do somatório dos valores atribuídos a cada parâmetro independentemente. Se o somatório dos valores for inferior a 40 pontos, este é classificado como impactado, de 41 a 60 como alterado e superior a 61 é considerado como área natural. Para maiores esclarecimentos sobre os atributos avaliados e sua respectiva pontuação consulte Callisto et al. (2002).

Com auxílio de um multianalisador (YSI Incorporated, mod. 85) obteve-se "in situ" os seguintes parâmetros da coluna d'água: temperatura, condutividade elétrica e oxigênio dissolvido. A determinação da alcalinidade total foi feita pelo método de Gram de acordo com Carmouze (1994), com titulação de ácido sulfúrico $0.01 \mathrm{~N}$.

\section{Analise estatística}

Uma Análise de Componentes Principais (Principal Components Analysis - PCA), baseada na matriz de correlação dos autovalores e autovetores, foi utilizada para ordenar os pontos de coleta com base nas variáveis abióticas (alcalinidade, temperatura, condutividade elétrica e oxigênio dissolvido), \%EPT, o protocolo de avaliação rápida e o índice $\mathrm{BMWP}-\mathrm{CETEC}$. A significância dos eixos foi medida segundo modelo de BrokenStick (Legendre \& Legendre 1998) e a diferença significativa entre os grupos (trechos alto, médio e baixo) foi feita através da analise ANOSIM, proposta por Clarke (1993), usando o índice de Gower e corrigida por Bonferroni (Hammer et al. 2001).

\section{RESULTADOS}

\section{Comunidade de macroinvertebrados bentônicos}

Um total de 17468 macroinvertebrados bentônicos foram coletados, distribuídos em 67 taxa (5 ordens e 57 famílias), durante os períodos amostrais. O filo Arthropoda 
apresentou maior abundância com cerca de $89 \%$ dos organismos coletados, seguido pelos filos Mollusca (6\%) e Annelida (5\%). Foram encontradas sete categorias taxonômicas: Hirudínea, Oligochaeta, Bivalvia, Gastropoda, Arachnoidea, Crustácea e Insecta (Figura 2). Dentre os organismos coletados, 924 foram identificados até o nível de classe, Como: Hirudínea, Oligochaeta, Bivalvia, Arachnoidea e Crustácea, apresentando 19, 21, 422, 413 e 49 indivíduos, respectivamente. A Classe Insecta apresentou maior número de indivíduos coletados (15839 indivíduos), seguida por Gastropoda (539 indivíduos). Além disso, Insecta também apresentou maior riqueza, sendo os organismos distribuídos em 52 famílias, nas ordens Odonata (5), Hemíptera (9), Coleoptera (9), Trichoptera (8), Diptera (10), Plecoptera (1), Collembola (1), Megaloptera (1), Ephemeroptera (7) e Lepidoptera (1). Na classe Gastropoda foram encontradas cinco famílias, representadas pelas ordens Basommatophora (2) e Mesogastropoda (3).

Dentre as ordens encontradas, Diptera foi a mais abundante, representando $37 \%$ dos indivíduos pertencentes à classe Insecta, com destaque para a família Chironomidae (88\% do total de Dípteros). As famílias Ceratopogonidae, Simullidae, Empididae, Tabanidae, Dytiscidae, Tipulidae, Stratiomyidae, Muscidae e Cullicidae também foram coletados, mas em baixa abundância (4\%, 3\%, 1\%, 0.1\%, 0.5\%, 1.5\%, 0.01\%, $0.01 \%$ e $0.1 \%$, respectivamente do total de Diptera). Na ordem Ephemeroptera também foi observada uma elevada abundância perfazendo $28 \%$ dos insetos, distribuídos nas famílias Leptohyphidae, Leptophlebiidae, Caenidae, Oligoneuriidae, Polymitarcyidae, Euthyplociidae e Baetidae. Por outro lado, Trichoptera e Plecoptera, apresentaram baixa abundância total de indivíduos coletados (7\% e 1\%, respectivamente). Apesar disso, para ordem Trichoptera foram encontrados elevados valores de riqueza dentro da bacia do rio Pandeiros. Os indivíduos desta ordem encontrados na bacia do rio Pandeiros estão distribuídos entre as famílias Odontoceridae, Helicopsychidae, Hydroptilidae, Polycentropodidae, Leptoceridae, Glossosomatidae, Philopotamidae e Hydropsychidae (Tabela 2 do Material Suplementar).

Os números totais de Ephemeroptera, Plecoptera e Trichoptera (EPT) foram maiores na bacia do rio Pandeiros na região média (2494, 19 e 445 indivíduos coletados, respectivamente), seguida pela alta $(1453,60$ e 537 indivíduos coletados, respectivamente), sendoquea regiãobaixa apresentou os menores valores (505, 2 e 110 indivíduos coletados respectivamente) (Figura 3). Quando analisado entre as três regiões as porcentagens de EPT separadamente para cada grupo taxonômico observou os maiores valores aferidos na porção média da bacia $(21.8 \%, 1.1 \%$ e $9.3 \%$, Ephemeroptera, Plecoptera e Trichoptera, respectivamente). Posteriormente, aparece à região alta $(27.8 \%, 0.2 \%$ e 5\%, Ephemeroptera, Plecoptera e Trichoptera, respectivamente), seguida da região baixa que apresentou os menores valores $(13.6 \%, 0.03 \%$ e $3.3 \%$, Ephemeroptera, Plecoptera e Trichoptera, respectivamente). Ao aferir ponto a ponto as porcentagens de EPT somadas, a região média apresentou o maior valor no P7 (52\% de EPT) e menor no P14 (17\% de EPT). Na região alta os maiores valores individuais foram observados nos pontos P1 e P4 (38\% de EPT em ambos) e o menor no P3 (24\% de EPT). A região baixa apresentou o maior valor no ponto P15 (36\%) e menor no P18 (3\%) (Tabela 1).

Outra ordem que apresentou elevado valor de riqueza foi Coleoptera, representando 17\% do total de indivíduos, nas famílias Dytiscidae, Gyrinidae, Hydrophilidae, Elmidae, Psephenidae, Scirtidae, Curculionidae,
Figura 2. Proporção dos indivíduos das principais classes (A) e das ordens pertencentes à classe insecta (B), encontradas na bacia do rio Pandeiros.

Figure 2. Proportion of individuals of the main classes (A) and insect orders belonging to the class (B), found in the Pandeiros river basin.

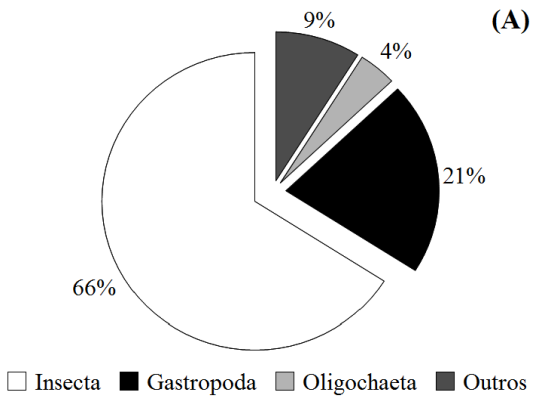

(A)

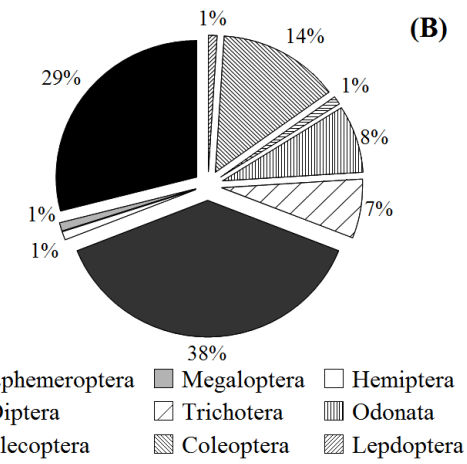




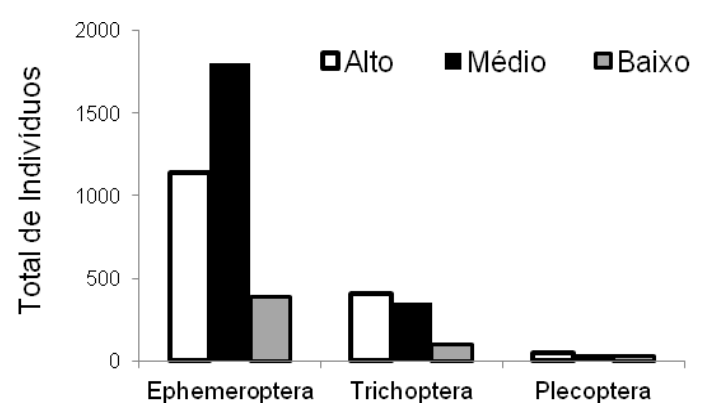

Figure 3. Total number of Ephemeroptera, Plecoptera and Trichoptera (EPT) collected in the upper (white), medium (black) and low (gray) regions of Pandeiros river basin.

Figura 3. Número total de Ephemeroptera, Trichoptera e Plecoptera (EPT) coletados nas regiões alta (branco), média (preto) e baixa (cinza) da bacia do rio Pandeiros.

Noteridae e Lutrochidae. As famílias Libellulidae, Gomphidae, Coenagrionidae, Calopterigidae e Aeshinidae, (Odonata), Naucoridae, Gerridae, Vellidae, Corixidae, Pleidae, Notonectidae, Mesovelidae e Hebridae, Belostomatidae (Hemíptera), Corydalidae (Megaloptera) e Pyralidae (Lepidoptera) foram encontradas na bacia do rio Pandeiros (Tabela 2 do Material Suplementar).

BWMP-CETEC, \%EPT, Protocolo de Avaliação Rápida e variáveis físicas e químicas de trecho de rio

O BWMP-CETEC apresentou os maiores valores nos trechos alto e médio da bacia. No trecho alto, os rios P4 e P2 apresentaram os maiores valores deste índice e P6 o menor. Na porção média da bacia quase todos os trechos apresentaram altos valores, principalmente o P7, P10 e o P8, enquanto nesta região o rio de menor pontuação foi o P13. A jusante, porção mais baixa e final da bacia, foi à região com maior número de trechos com baixa pontuação, como P18 e P19. O trecho de maior pontuação nesta região foi o P15 (Tabela 1). O Protocolo de Avaliação Rápida na região de cabeceira apresentou os maiores valores nos trechos do rio P1, P3 e P4. O P6, localizado na região alta da bacia apresentou o menor valor de todos os trechos avaliados. Os trechos do rio P10 e P11 apresentaram as

Tabela 1. Valores médios e erro padrão da porcentagem de oxigênio dissolvido na água $\left(\mathrm{O}_{2}\right)$, condutividade elétrica $(\mu \mathrm{S} / \mathrm{cm})$, temperatura da água ${ }^{\circ} \mathrm{C}$ (temp.), alcalinidade $(\mu \mathrm{Eq} / \mathrm{L})$, do índice BMWP-CETEC (Junqueira \& Campos 1998), Protocolo de Avaliação Rápida (PAR) (Callisto et al. 2002) e porcentagem de Ephemeroptera, Plecoptera e Trichoptera (EPT), representando a condição ambiental dos trechos estudados nos pontos amostrais nas regiões alta, média e baixa da bacia do rio Pandeiros. Ordem dos rios segundo Strahler (1963).

Table 1. Values, mean values and standard error of the percentage of dissolved oxygen in water $\left(\mathrm{O}_{2}\right.$ Sat $\left.\%\right)$, electrical conductivity $(\mu \mathrm{S} / \mathrm{cm})$, water temperature $\left(\right.$ temp. $\left.{ }^{\circ} \mathrm{C}\right)$, alkalinity $(\mu \mathrm{Eq} / \mathrm{L})$, of the index BMWP-CETEC (Junqueira \& Campos 1998), the Fast Assessment Protocol (PAR) (Callisto et al. 2002) and percentage of Ephemeroptera, Plecoptera e Trichoptera (\%EPT) representing the environmental condition of the sites studied in the sampling points in the regions upper, middle and lower Pandeiros river basin. Order of the river by Strahler (1963).

\begin{tabular}{|c|c|c|c|c|c|c|c|c|c|c|}
\hline Região & Pontos & Rio & $\mathrm{O} 2$ & Condutividade & Temp. & Alcalinidade & $\begin{array}{l}\text { BMWP- } \\
\text { CETEC }\end{array}$ & PAR & EPT & Ordem \\
\hline \multirow{6}{*}{$\frac{\pi}{4}$} & 1 & Pandeiros -T1 & $73 \pm 25$ & $65 \pm 5$ & $24.1 \pm 1.2$ & $395 \pm 254$ & 149 & 92 & 38.5 & 1 \\
\hline & 2 & São Pedro & $80 \pm 4$ & $182 \pm 17$ & $23.1 \pm 0.9$ & $757 \pm 514$ & 175 & 90 & 29.9 & 3 \\
\hline & 3 & Salobro & $63 \pm 21$ & $64 \pm 1$ & $26.8 \pm 0.1$ & $236 \pm 184$ & 147 & 94 & 24.5 & 1 \\
\hline & 4 & Panelas & $73 \pm 13$ & $93 \pm 57$ & $21.4 \pm 2$ & $204 \pm 296$ & 191 & 92 & 38.7 & 1 \\
\hline & 5 & São Domingos & $70 \pm 19$ & $42 \pm 23$ & $22.5 \pm 2.4$ & $179 \pm 137$ & 142 & 80 & 26.4 & 4 \\
\hline & 6 & Pandeiros -T2 & $66 \pm 25$ & $76 \pm 7$ & $25 \pm 1$ & $458 \pm 206$ & 82 & 60 & 36.4 & 4 \\
\hline \multirow{8}{*}{$\frac{\pi}{\stackrel{\pi}{0}}$} & 7 & Catolé & $67 \pm 27$ & $38 \pm 50$ & $21.9 \pm 1.9$ & $734 \pm 915$ & 164 & 86 & 52.1 & 4 \\
\hline & 8 & Pindaibal & $40 \pm 27$ & $27 \pm 19$ & $24.8 \pm 1.6$ & $164 \pm 295$ & 157 & 90 & 44.2 & 3 \\
\hline & 9 & Alegre & $40 \pm 4$ & $28 \pm 38$ & $23.3 \pm 1.9$ & $130 \pm 186$ & 127 & 80 & 35.3 & 3 \\
\hline & 10 & Pandeiros -T3 & $72 \pm 25$ & $60 \pm 2$ & $23.7 \pm 1.4$ & $469 \pm 197$ & 159 & 94 & 34.3 & 5 \\
\hline & 11 & Pandeiros -T4 & $73 \pm 23$ & $60 \pm 2$ & $23.3 \pm 1.4$ & $374 \pm 215$ & 143 & 94 & 44.1 & 5 \\
\hline & 12 & Pandeiros -T5 & $73 \pm 25$ & $60 \pm 3$ & $23.3 \pm 1.7$ & $494 \pm 156$ & 138 & 90 & 18.7 & 5 \\
\hline & 13 & Borrachudo & $49 \pm 30$ & $77 \pm 18$ & $19.8 \pm 2.2$ & $526 \pm 83$ & 83 & 60 & 19.4 & 3 \\
\hline & 14 & Macaúba & $34 \pm 25$ & $277 \pm 6$ & $21.8 \pm 1.5$ & $2590 \pm 1340$ & 95 & 90 & 17.3 & 3 \\
\hline \multirow{6}{*}{ 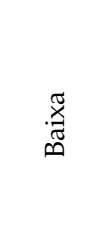 } & 15 & Pandeiros -T6 & $70 \pm 29$ & $105 \pm 5$ & $25.6 \pm 0.1$ & $1178 \pm 219$ & 156 & 86 & 36.8 & 5 \\
\hline & 16 & Pandeiros -T7 & $81 \pm 15$ & $71 \pm 4$ & $25.1 \pm 2.4$ & $1324 \pm 1680$ & 113 & 90 & 6.7 & 5 \\
\hline & 17 & Pandeiros -T8 & $71 \pm 24$ & $72 \pm 2$ & $25.9 \pm 2.4$ & $486 \pm 120$ & 126 & 86 & 17 & 5 \\
\hline & 18 & Pandeiros -T9 & $48 \pm 41$ & $82 \pm 11$ & $29.1 \pm 5.9$ & $510 \pm 273$ & 42 & 46 & 3.2 & 5 \\
\hline & 19 & Pandeiros -T10 & $71 \pm 24$ & $70 \pm 5$ & $25 \pm 1.9$ & $605 \pm 284$ & 78 & 70 & 15.5 & 5 \\
\hline & 20 & Pandeiros -T11 & $45 \pm 28$ & $71 \pm 5$ & $26.2 \pm 2.2$ & $1298 \pm 1226$ & 90 & 65 & 23.1 & 5 \\
\hline \multirow{3}{*}{\multicolumn{2}{|c|}{ Valores Médios }} & Trecho Alto & $71 \pm 2$ & $78 \pm 4$ & $23.8 \pm 1.4$ & $372 \pm 15$ & $84.7 \pm 5$ & 147.7 & $2.4 \pm 2.5$ & \\
\hline & & Trecho Médio & $56 \pm 4$ & $79 \pm 9$ & $22.7 \pm 1.2$ & $685 \pm 28$ & $85.7 \pm 4$ & $133.3=$ & $3.2 \pm 4.7$ & \\
\hline & & Trecho Baixo & $50 \pm 4$ & $87 \pm 7$ & $26.1 \pm 1.2$ & $900 \pm 20$ & $73.8 \pm 7$ & $100.8 \pm 1$ & $7.1 \pm 4.9$ & \\
\hline
\end{tabular}


Tabela 2. Valores de correlação e coeficiente dos eixos 1 e 2 na analise PCA da porcentagem de oxigênio dissolvido na água $\left(\mathrm{O}_{2}\right)$, condutividade elétrica $(\mu \mathrm{S} / \mathrm{cm})$, temperatura da água ${ }^{\circ} \mathrm{C}$ (temp.), alcalinidade $(\mu \mathrm{Eq} / \mathrm{L})$, Protocolo de Avaliação Rápida (Callisto et al. 2002), porcentagem de Ephemeroptera, Plecoptera e Trichoptera (EPT) e índice BMWPCETEC (Junqueira \& Campos 1998).

Table 2. Correlation coefficient values and axes 1 and 2 in the PCA analysis of the percentage of dissolved oxygen in water $\left(\mathrm{O}_{2}\right)$, electrical conductivity $(\mu \mathrm{S} / \mathrm{cm})$, water temperature in ${ }^{\circ} \mathrm{C}$ (temp.), alkalinity $(\mu \mathrm{Eq} / \mathrm{L})$, the Fast Assessment Protocol (Callisto et al. 2002), percentage of Ephemeroptera, Plecoptera e Trichoptera (EPT) and BMWP index-CETEC (Junqueira \& Fields 1998).

\begin{tabular}{lccccccc}
\hline & $\mathrm{O}_{2}$ & Condutividade & Temp. & Alcalinidade & Protocolo & BMWP-CETEC & EPT \\
\hline Correlação (Eixo 1) & 0.39 & -0.3 & -0.39 & -0.34 & 0.83 & 0.85 & 0.68 \\
Correlação (Eixo 2) & 0.05 & 0.79 & -0.47 & 0.79 & 0.05 & 0.19 & 0.14 \\
Coeficientes (Eixo 1) & 0.25 & -0.19 & -0.25 & -0.22 & 0.53 & 0.55 & 0.43 \\
Coeficientes (Eixo 2) & 0.04 & 0.63 & -0.38 & 0.63 & 0.04 & 0.15 & 0.11 \\
\hline
\end{tabular}

maiores pontuações na porção média da bacia, enquanto o rio P13 apresentou o menor valor desta região da bacia. Na região a jusante o rio P16 apresentou o maior valor, enquanto o rio P18 apresentou o menor valor deste protocolo (Tabela 1).

A Análise ANOSIM $(\mathrm{R}=0.28 ; P<0.001)$ mostrou que os trechos alto $(P<0.0003)$ e médio $(\mathrm{P}=0.0043)$ da bacia são significativamente diferentes da porção a jusante pela análise de Componentes Principais baseada nas variáveis ambientais (oxigênio dissolvido, condutividade elétrica, temperatura e alcalinidade da água), Protocolo de Avaliação Rápida e BMWP-CETEC (Figura 4; Tabela 2). A variância total das métricas ambientais estudadas foi de $56.4 \%$ sendo que o primeiro e o segundo eixos explicaram $34.3 \%$ e $22.1 \%$ da variância, respectivamente (onde ambos foram significativos segundo Broken-Stick). Alcalinidade e condutividade se correlacionaram negativamente ao eixo 1 positivamente ao eixo 2 . A temperatura correlacionou negativamente ao eixo 1 e 2 , enquanto o oxigênio dissolvido, \%EPT, BMWP-CETEC e o Protocolo de avaliação rápida positivamente a ambos os eixos. Os coeficientes mais altos do primeiro eixo, que agrega maior variância, foram observados no BMWP-CETEC, \%EPT e no Protocolo de Avaliação Rápida (Figura 4; Tabela 2).

\section{DISCUSSÃO}

Neste trabalho destaca-se devido os resultados indicarem a viabilidade da aplicação das métricas avaliadas em outros sistemas para o bioma cerrado. O fato do maior número de espécimes encontrados pertencerem ao filo Arthropoda era esperado, pois este possui a maior diversidade estimada de espécies tanto no Brasil (88790 a 118290 espécies) quanto no mundo (1077200 a 1097400 espécies) (Lewinsohn \& Prado 2005). Este filo tem representantes em todos os ambientes aquáticos, sejam eles pequenos como uma poça de água ou copos de bromélia, até em

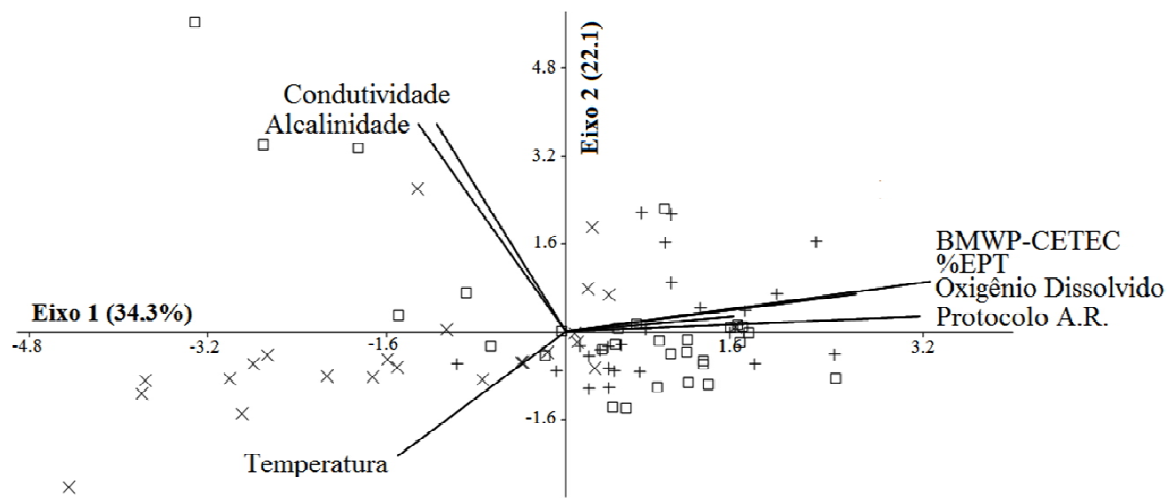

Figure 4. Distribution of points in the principal components analysis based on environmental variables (conductivity, alkalinity, temperature, dissolved oxygen), percentage of Ephemeroptera, Plecoptera e Trichoptera (\%EPT) index BMWP-CETEC (Junqueira \& Campos 1998) and Fast Assessment Protocol (Callisto et al. 2002), the upper stretches (cross), medium (square) and low (crux). Values of the variance of the first and second axis.

Figura 4. Distribuição dos pontos na analise de Componentes Principais baseada nas variáveis ambientais (condutividade, alcalinidade, temperatura, oxigênio dissolvido), porcentagem de Ephemeroptera, Plecoptera e Trichoptera (\%EPT) índice BMWP-CETEC (Junqueira \& Campos 1998) e protocolo de avaliação rápida (Callisto et al. 2002), dos trechos alto (cruz), médio (quadrado) e baixo (xis). Valores de variância do primeiro e o segundo eixo. 
grandes rios e lagos (Lewinsohn \& Prado 2002; Rezende et al. 2010; Gonçalves et al. 2012). A classe Insecta apresentou maior número de indivíduos coletados e foi a mais rica, como observado em outros trabalhos (Ribeiro \& Uieda 2005). Comparando aos resultados encontrados por Moreno \& Calisto (2005) estudando áreas de referência da Bacia do Rio das Velhas, também pertencente à Bacia do Rio São Francisco, a elevada quantidade de taxa encontrada em nosso estudo indica um ambiente preservado (65 e 67 taxa, para as Bacias dos Rios Velhas e Pandeiros, respectivamente). Em ambientes degradados, geralmente são encontrados poucos taxa na comunidade bentônica, porém com elevadas densidades. Assim, é importante encontrar uma ampla variedade de organismos para atestar a saúde ambiental do corpo aquático, desde os sensíveis à poluição até os tolerantes e resistentes (Rosenberg \& Resh 1993; Roldán 2001). Esta informação é importante, pois evidência o elevado grau de preservação do ecossistema em relação a outros pertencentes à Bacia Hidrográfica do Rio São Francisco, demonstrando a potencialidade do mesmo para ser utilizado como área de referência para a os corpos aquáticos da Bacia do alto Rio São Francisco, onde não há estudos semelhantes.

A elevada abundância de Diptera dentro da comunidade de invertebrados aquáticos coletados neste estudo deve-se à família Chironomidae. Segundo Merrit \& Cummins (1996), Chironomidae é um dos grupos mais abundantes de insetos aquáticos, e que geralmente compõem mais de $80 \%$ da entomofauna, tanto em ambiente lêntico como lótico, assim como observado neste estudo. Chironomidae é um grupo importante, pois participa como um elo fundamental em várias teias alimentares (Nolte 1986). Segundo Burki et al. (1978), alguns representantes podem desenvolver resistência ou se adaptar (quanto a sua fisiologia ou comportamento) às diversas condições do meio. Além disso, podem apresentar uma elevada diversidade de espécies e uma estratégia trófica generalista (Gonçalves et al. 2003; Callisto et al. 2007). Estes aspectos associados poderiam explicar o fato destes organismos serem abundantes não apenas na bacia do rio Pandeiros, mas também em outros locais como relatado por Ruaro et al. (2010) na avaliação da qualidade da água do rio Clarito.

A elevada abundância e porcentagem de Ephemeroptera na comunidade bentônica e a alta riqueza de Trichoptera dentro da mesma podem ser um indicador de boa qualidade da água na bacia do Rio Pandeiros (Moreno \& Callisto 2005; Rosenberg \& Resh 1993; Roldán 2001). Assim, a região apresenta-se preservada, provavelmente, devido à baixa densidade populacional humana, baixa descarga de efluentes nos corpos aquáticos, poucos pontos de erosão e presença de matas ciliares bem preservadas (Fonseca et al. 2008). Os EPT são considerados excelentes indicadores ambientais, sendo as famílias encontradas no presente estudo, sensíveis às alterações ambientais, partindo de premissas do índice BMWP (Moreno \& Callisto 2005; Junqueira \& Campos 1998; Ruaro et al. 2010; Galdean et al. 2000). Isto se deve ao fato destes organismos requererem elevadas concentrações de oxigênio dissolvido na água, por possuírem pouca tolerância a poluentes, como também corroborado pela associação destes dois fatores pela PCA. Além disso, estes indicadores são encontrados em ambientes com alta diversidade de hábitats e microhábitats, sendo bioindicadores importantes na avaliação da qualidade de água e, sobretudo de qualidade ambiental (Sandin \& Johnson 2000).

A \%EPT, o BMWP-CETEC, Protocolo de Avaliação Rápida e os elevados valores de oxigênio dissolvido na água indicaram boa qualidade das águas, principalmente, nos trechos alto e médio da bacia do rio Pandeiros, corroborado os resultados de abundância e riqueza encontrados para os macroinvertebrados. Porém, Monteiro et al. (2008) estudando a bacia do rio Meia Ponte (GO), observou os melhores resultados a jusante (parte com menor densidade demográfica), devido provavelmente, a um processo de autodepuração natural do rio. Estes padrões observados nos trechos altos e médios corroboram os resultados da analise ANOSIM, que revelou também que estes trechos são similares quanto a suas características (físicas e químicas da água, \%EPT, protocolo de avaliação rápida e índice BMWP-CETEC). Os coeficientes mais altos do primeiro eixo, que agrega maior variância, foram observados na \%EPT, BMWPCETEC e no Protocolo de Avaliação Rápida, indicando que estes parâmetros foram os mais importantes para agregar os grupos estudados (Clarke 1993) e informando que estes são uma ferramenta importante na avaliação ambiental na região de estudo.

Os dados indicaram uma diminuição gradual nos valores de BMWP-CETEC ao longo dos 
trechos estudados na Bacia do Rio Pandeiros, de montante para jusante, em direção à foz com o Rio São Francisco. Apesar disso, na parte baixa da bacia foi observado trecho com água de qualidade satisfatória, índice mais baixo deste trabalho. Este fato pode ser associado a uma maior pressão antrópica, sobre a vegetação ripária da bacia do rio Pandeiros como relatada por Fonseca et al. (2008). Esta pressão é decorrente principalmente da utilização de plantas lenhosas para produção de carvão e fins madeireiros, e pode ser responsável pela maior temperatura da água neste trecho em função da maior exposição à radiação solar. Além disso, no trecho final da bacia ocorre elevada atividade de urbanização, causando assim maior impacto quando comparado ao restante da bacia (Fonseca et al. 2008). Este fato pode ser também decorrente de um saneamento básico inexistente ou precário, agravando-se principalmente no vilarejo de Pandeiros, área de maior concentração populacional da região. Isso é corroborado também pelos elevados valores de condutividade elétrica e alcalinidade, que frequentemente estão associados à maior atividade antrópica (Gafner \& Robinson 2007; Ometo et al. 2000).

Os resultados encontrados na parte à jusante da bacia também mostraram baixa abundância e porcentagem de EPT, como observado também por Lopes et al. (2008) em sistemas amazônicos. Estas alterações na abundância relativa, principalmente de Ephemeroptera, evidência efeitos da degradação ambiental sobre a biota em algumas áreas da bacia do rio Pandeiros, possivelmente pela maior urbanização, assim como também observado por Ruaro et al. (2010). Outro aspecto importante e muito comum na região é o impacto da agricultura e pecuária, que também se intensifica na porção final da bacia (Fonseca et al. 2008). Esta pode constituir uma elevada fonte de poluição para os rios, como demonstrado por Merten \& Minella (2002), que constatou que a ocupação e uso do solo pelas atividades agropecuárias alteram sensivelmente os processos biológicos, físicos e químicos dos sistemas naturais (Yung-Chul et al. 2011). Este somatório de fatores altera as condições naturais do corpo aquático (Ometo et al. 2000), e favorecendo a poucos organismos tolerantes a esta condição adversa, justificando a mudança na comunidade de macroinvertebrados nestes trechos (Roy et al. 2003). Mas, apesar de apresentar pontos de alteração antrópica à jusante da bacia, a qualidade da água ainda é apontada como ótima na maioria dos trechos avaliados. Este fato ocorre devido às características climáticas, geomorfológica e vegetacionaís (zona de transição entre Cerrado e Caatinga), que dificultam a utilização da região para agricultura e povoamento, fazendo com que ainda não se observe elevados níveis de degradação neste local (Silva \& Bates 2002).

Através dos resultados concluímos que a bacia do rio Pandeiros possui, de forma geral, águas de ótima qualidade. Esta apresenta uma elevada diversidade de macroinvertebrados bentônicos se comparada a outras do rio São Francisco (e.g., Rio das Velhas), indicando elevada preservação ambiental. Nos trechos à jusante da bacia, em direção ao pântano, foram observados baixos valores nos parâmetros avaliados de qualidade ambiental, refletindo sobre a comunidade de macroinvertebrados bentônicos. Isso ocorre principalmente, devido à atividade agropecuária e sua expansão, além do fato de ser a região mais urbanizada, necessitando maiores cuidados nesta parte da Bacia do Rio Pandeiros. Outro aspecto importante é que os resultados indicam a aplicação das métricas avaliadas em outros sistemas do bioma cerrado.

Agradecimento: Ao CNPq pelo financiamento ao projeto "Ecologia e Biodiversidade de Macrófitas e Invertebrados Aquáticos do Refúgio Biológico do Rio Pandeiros/MG", segundo o edital MCT/CNPq/CT-Hidro - No 555488/ 2006-3, possibilitando a realização deste manuscrito, e também ao IEF (Instituto Estadual de Florestas), pelo apoio logístico no transporte e alojamento nos períodos de coleta. Agradecemos também aos dois revisores anônimos que muito contribuíram para melhora deste artigo.

\section{BIBLIOGRAFIA}

ALLAN, JD. 2004. Landscapes and riverscapes: the influence of land-use on stream ecosystems. Annu. Rev. Ecol. Evol. Syst., 35:257-84.

BEEBY, A. 1993. Applying Ecology. London: Chapman \& Hall.

BURKI, E; R ROTHEN \& A ScHOLL. 1978. Koexstenz von zwei cytolgisch verschiedenen Populationen der zuckmucke Chironomus plumosos im Murtensee. Rev. Suisse Zoo., 85:625-634.

Callisto, M \& JFGJr Gonçalves. 2005. Bioindicadores bentônicos. Pp. 371-379 in: Roland F; D César \& M Marinho. Lições de limnologia.

Callisto, M \& P Moreno. 2008. Programa de biomonitoramento de qualidade de água e diodiversidade bentônica na bacia do Rio das Velhas. Projeto Manuelzão: a história da mobilização que começou em torno de um rio, 1:107-121.

Callisto, M; JFGJr Gonçalves \& M Graca. 2007. Leaf litter as a possible food source for chironomids in headwater streams. Rev. Bras. Zool., 24:442-448.

Callisto, M; W Ferreira; P Moreno; MDC Goulart \& M Petrucio. 2002. Aplicação de um protocolo de avaliação rápida da diversidade de habitats em atividades de ensino 
e pesquisa (MG-RJ). Acta Limnol. Bras., 14:91-98.

Carmouze, JP. 1994. O Metabolismo dos Ecossistemas Aquáticos. Fundamentos teóricos, métodos de estudo e análises químicas. São Paulo: Edgard Blücher/FAPESP. Pp. 253.

CLARKE, KR. 1993. Non-parametric multivariate analyses of changes in community structure. Aust. J. Ecol., 18:117-43.

Cortes, R; S Varandas; A Teixeira; SJ Hughes; J Magalhâes; ET AL. 2011. Effects of landscape metrics and land-use variables on macroinvertebrate communities and habitat characteristics. Limnetica, 30:347-362

DOLÉDEC, S; N PHILLIPS \& C TOWNSEND. 2011. Invertebrate community responses to land use at a broad spatial scale: trait and taxonomic measures compared in New Zealand rivers. Freshwater Biol., 56:1670-1688.

Dudgeon, D; AH Arthington; MO Gessner; ZI KaWABata; DJ KNOWLER; ET AL. 2006. Freshwater biodiversity: importance, threats, status and conservation challenges. Biol. Review, 81:163-1826.

Ferreira, WR; LT Paiva \& M Callisto. 2011. Development of a benthic multimetric index for biomonitoring of a neotropical watershed. Braz. J. Biol., 71:15-25.

FONSECA, EMB; WR Grossi; RA FIoRINE \& NJS PRADO. 2008. PCH Pandeiros: uma complexa interface com a gestão ambiental regional. VI Simpósio Brasileiro Sobre Pequenas e Médias Centrais Hidrelétricas. Belo Horizonte - MG.

Gafner, C \& CT Robinson. 2007. Nutrient enrichment influences the responses of stream macroinvertebrates to disturbance. J. N. Am. Benthol. Soc., 26:92-102.

Galdean, N; M Callisto \& FAR Barbosa. 2000. Lotic Ecosystems of Serra do Cipó, southeast Brazil: water quality and a tentative classification based on the benthic macroinvertebrate community. Aquat. Ecosys. Health Manage., 3:545-552.

Gonçalves, JFJr; FA Esteves \& M Callisto. 2003. Chironomids colonization in Nymphaea ampla L. detritus during a degradative ecological succession experiment in a Brazilian coastal lagoon. Acta Limnol. Bras., 15:1-27.

Gonçalves, JFJr; RS Rezende; J França \& M Callisto. 2012. Invertebrate colonisation during leaf processing of native, exotic and artificial detritus in a tropical stream. Mar. Freshwat. Res. 63:428-439.

Goulart, M \& M Callisto. 2003. Bioindicadores de qualidade de água como ferramenta em estudos de impacto ambiental. Rev. FAPAM, 2:1-22.

Gualdoni, CM; CA Duarte \& EA MEdeot. 2011. Ecological status of two mountain streams of the south of Cordoba, Argentina. Ecol. Austral, 21:149-162.

Hammer, Ø; DAT Harper \& PD RYAn. 2001. PAST: Paleontological Statistics Software Package for Education and Data Analysis. Palaeont. Electronica, 4:1-9.

HAWKs, HA. 1997. Origin and development of the Biological Monitoring Working Party system. Water Research, 32:964-968.

JUNQUEIRA, VM \& SCM CAMPOS. 1998. Adaptation of the "BWMP" Method for Water Quality Evaluation to rio das Velhas watershed (Minas Gerais, Brazil). Acta Limnol. Bras., 10:125-135.

JunQueIRA, VM \& SCM CAMPos. 1998. Adaptation of the "BWMP" Method for Water Quality Evaluation to rio das Velhas watershed (Minas Gerais, Brazil). Acta Limnol. Bras., 10:125-135.

LEGENDRE, P \& L LEGENDRE. 1998. Numerical Ecology. English Ed. Elsevier.

LeWInsohn, TM \& PI Prado. 2002. Biodiversidade brasileira: sintese do estado do conhecimento atual. São Paulo: Contexto
Acadêmica. Pp. 139-144.

LeWInsohn, TM \& PI Prado. 2005. Quantas especies há no Brasil? Megadiversidade, 1:36-51.

LOPES, MJN; MSR SILVA; RTM SAMPAIO; ELL BELMONT \& CS SANTOS-NETO. 2008. Avaliação preliminar da qualidade da água de bacias hidrográficas de Manaus utilizando o método BMWP adaptado. SaBios, 3:1-9.

Magnusson, WE. 2001. Time in Hydrology. Facets of Hydrology II. New York:. IN Rodda. Pp. 249-290.

Merrit, RW \& KW Cummins. 1996. An introduction to the aquatic insects of North America. 3rd edition. Dubuque: Kendall/Hunt Publishing Company. Pp. 862.

Merten, GH \& JP MinelLa. 2002. Qualidade da água em Bacias Hidrográficas rurais: um desafi o atual para a sobrevivência futura. Agroecol. Desenv. Rural Sustent., 3:33-38.

Metzeling, L; B Chessman; R Hardwick \& V Wong. 2003 Rapid assessment of rivers using macroinvertebrates: the role of experience, and comparisons with quantitative methods. Hydrobiologia, 510:39-52.

Monteiro, TR; LG Oliveira \& BS Godoy. 2008. Biomonitoramento da qualidade de água utilizando macroinvertebrados bentônicos: adaptação do índice biótico BMWP à bacia do rio Meia Ponte-GO. Oecol. Bras., 12:553-563.

Moreno, P; M Callisto. 2005. Bioindicadores de qualidade de água ao longo da Bacia do Rio das Velhas (MG). In: Bioindicadores de qualidade de água. 5a ed. Brasília: Embrapa. Pp. 95-116.

Morgan, F; AM OliveIra \& M Callisto. 2006. Inventário da diversidade de macroinvertebrados bentônicos no reservatório da estação ambiental de Peti, MG, Brasil. Neotrop. Biol. Cons., 1:17-23.

Nessimian, JL; EM Venticingue; J Zuanon; P De Marco; M GORDO; ET AL. 2008. Land use, habitat integrity, and aquatic insect assemblages in Central Amazonian streams. Hydrobiologia, 614:117-131.

Nolte, U. 1986. Erstbesiedlung kunstlicher kleingewasser und primare Sukzession des Makrozoobenthos unter besonderer Berucksichtigung der Chironomidae (Diptera) Feldexperimente in Zentralamazonien. Mathematisch-Tese de Doutorado. Naturwissenschaflichen Fachbereiche der Georg-Universitat zu Gottingen.

Ometo, JP; LA Martinelli; MV Ballester; A Gessner; V KRUSCHE; ET AL. 2000. Effects of land use on water chemistry and macroinvertebrates in two streams of the Piracicaba river basin, Southeast Brazil. Freshwater Biol., 44:327-337.

PAZ, A;P MORENO; L ROCHA \& M CALlisto. 2008. Efetividade de Áreas Protegidas na conservação da qualidade das águas e biodiversidade aquática em sub-bacias de referência no Rio das Velhas (MG). Neotrop. Biol. Cons., 3:149-158.

Rezende, RS; JfJR Gonçalves \& MM Petrucio. 2010. Leaf breakdown and invertebrate colonization of Eucalyptus grandis (Myrtaceae) and Hirtella glandulosa (Chrysobalanaceae) in two Neotropical lakes. Acta Limnol. Bras., 22:23-34.

RIBEIRO, LO \& VS UIEDA. 2005. Estrutura da comunidade de macroinvertebrados bentônicos de um riacho de Serra do Itatinga, São Paulo, Brasil. Rev. Bras. Zoo., 22:613-618.

Roldán, GP. 1988. Guía para el estudio de los macroinvertebrados acuáticos del Departamento de Antioquia. Bogotá: Fondo Fen Colombia, Colciências, Ed. Universidad de Antioquia.

RoLDÁN, GP. 2001. Los macroinvertebrados acuáticos y su uso como indicadores de la calidad del agua. Editorial Presencia, 
Bogotá. Colombia.

ROSENBERG, DM \& VH ReSH. 1993. Freshwater biomonitoring and benthic macroinvertebrates. New York: Chapman \& Hall. Pp. 488.

Roy, AH; AD Rosemond; MJ PaUl; DS Leigh \& JB WALLACE. 2003. Stream macroinvertebrate response to catchment urbanisation (Georgia, U.S.A.). Freshwater Biol., 48:329-346.

Ruaro, R; M Agustini \& F Orssatto. 2010. Avaliação da qualidade da água do rio Clarito no município de Cascavel (PR), através do índice BMWP adaptado. SaBios, 5:1-08.

SANDIN, L \& RK JoHNSON. 2000. The statistical power of selected indicator metrics using macroinvertebrates for assessing acidification and eutrophication of running waters. Hydrobiologia, 422:233-243.

SANDIN, L \& RK JoHNSON. 2000. The statistical power of selected indicator metrics using macroinvertebrates for assessing acidification and eutrophication of running waters. Hydrobiologia, 422:233-243,

SCRImGeOUR GJ \& S Kendall. 2003. Effects of livestock grazing on benthic invertebrates from a native grassland ecosystem. Freshwater Biol., 48:347-62.

Silva, JMC \& JM Bates. 2002. Biogeographic patterns and conservation in the South American cerrado: a tropical savanna hotspot. BioScience, 52:225-233.

SMITH, MJ;WRKAY;DHDEDWARD;PJPAPAS; KRICHARDSONM; ET AL. 1999. AusRivAS: using macroinvertebrates to assess ecological condition of rivers in Western Australia. Freshwater Biology, 41:269-282.

Stark, JD; I Boothroyd; J Harding; J Maxted \& M SCARSBROOK. 2001. Protocols for Sampling Macroinvertebrates in Wadeable Streams. New Zealand Macroinvertebrate Working Group, Report No. 1. Prepared for the Ministry for the Environment. Sustainable Management Fund Project No. 5103. Pp. 57. www.caw thron.org.nz/coastalfreshwater-resources/downloads. html. Last accessed: January 3rd, 2012.

Stoddard, JL; DV PeCK; SG PAulsen; J VAN-Sickle; CP HaWKINS; ET AL. 2005. An ecological assessment of western streams and rivers. EPA 620/R-05/005, U.S. Washington, DC: Environmental Protection Agency.

Strahler, AN. 1963. The Earth Sciences. Harper \& Row, New York, NY.

Yung-Chul, J; K Nan-Young; K SoOn-JoK; H Seung-Chul; H IN-Chul; ET AL. 2011. Effects of land use on benthic macroinvertebratecommunities: Comparison of twomountain streams in Korea. Ann. Limnol. - Int. J. Lim., 47:35-49. 


\section{Material Suplementar}

Tabela 1. Base hidrográfica Otto-codificada e localização geografica dos rios (UTM). Em negrito o nivel de otto-bacia (Otto5) utilizado na segregação das regiões.

Table 1. Base watershed Otto-coded and geographical location of the river (UTM). Bold the level of otto-bacia (Otto5) used in segregation of the regions.

\begin{tabular}{ccccccccccc}
\hline Região & Pontos & Otto1 & Otto2 & Otto3 & Otto4 & Otto5 & Otto6 & Lat_Sad69 & Long-Sad69 & Localização Geográfica - UTM \\
\hline & P1 & 7 & 74 & 747 & 7472 & $\mathbf{7 4 7 2 9}$ & 747291 & -15.185 & -45.124 & 23 L 487078 8321388 \\
A & P2 & 7 & 74 & 747 & 7472 & $\mathbf{7 4 7 2 8}$ & 747281 & -15.222 & -45.139 & 23 L 485084 8317123 \\
L & P3 & 7 & 74 & 747 & 7472 & 74727 & 747279 & -15.255 & -45.087 & 23 L 490766 8313432 \\
T & P4 & 7 & 74 & 747 & 7472 & 74727 & 747274 & -15.257 & -44.963 & 23 L 505376 8312902 \\
A & P5 & 7 & 74 & 747 & 7472 & 74727 & 747276 & -15.262 & -45.014 & 23 L 498526 8312719 \\
& P6 & 7 & 74 & 747 & 7472 & 74727 & 747275 & -15.282 & -45.013 & 23 L 498679 8310507 \\
& P7 & 7 & 74 & 747 & 7472 & 74726 & 747263 & -15.29 & -44.822 & 23 L 519132 8309678 \\
& P8 & 7 & 74 & 747 & 7472 & $\mathbf{7 4 7 2 6}$ & 747262 & -15.384 & -44.945 & 23 L 505963 8299244 \\
M & P9 & 7 & 74 & 747 & 7472 & 74725 & 747252 & -15.457 & -44.859 & 23 L 515210 8291122 \\
E & P10 & 7 & 74 & 747 & 7472 & 74725 & 747251 & -15.441 & -44.822 & 23 L 519165 8292960 \\
D & P11 & 7 & 74 & 747 & 7472 & 74723 & 747231 & -15.456 & -44.789 & 23 L 522615 8291137 \\
I & P12 & 7 & 74 & 747 & 7472 & 74723 & 747232 & -15.455 & -44.79 & 23 L 5226798291483 \\
A & P13 & 7 & 74 & 747 & 7472 & 74724 & 747241 & -15.423 & -44.789 & 23 L 522734 8294876 \\
& P14 & 7 & 74 & 747 & 7472 & $\mathbf{7 4 7 2 2}$ & 747221 & -15.477 & -44.744 & 23 L 527480 8288900 \\
& P15 & 7 & 74 & 747 & 7472 & $\mathbf{7 4 7 2 1}$ & 747212 & -15.514 & -44.754 & 23 L 526443 8284887 \\
B & P16 & 7 & 74 & 747 & 7472 & $\mathbf{7 4 7 2 1}$ & 747212 & -15.519 & -44.754 & 23 L 526523 8284205 \\
A & P17 & 7 & 74 & 747 & 7472 & 74721 & 747211 & -15.606 & -44.711 & 23 L 531023 8274679 \\
I & P18 & 7 & 74 & 747 & 7472 & 74721 & 747211 & -15.667 & -44.635 & 23 L 539212 8267918 \\
X & P19 & 7 & 74 & 747 & 7472 & 74721 & 747211 & -15.667 & -44.639 & 23 L 538754 8267922 \\
A & P20 & 7 & 74 & 747 & 7472 & $\mathbf{7 4 7 2 1}$ & 747211 & -15.683 & -44.61 & 23 L 541841 8266150 \\
\hline
\end{tabular}

Tabela 2. Ocorrência de macroinvertebrados bentônicos nos pontos amostrais coletados nos meses de fevereiro, maio, setembro e novembro de 2008, ao longo da bacia do rio Pandeiros.

Table 2. Occurrence of macroinvertebrates benthic collected in sampling sites in February, May, September and November 2008, along the Pandeiros river basin.

\begin{tabular}{|c|c|c|c|c|c|c|c|c|c|c|c|c|c|c|c|c|c|c|c|c|}
\hline \multirow{2}{*}{\multicolumn{21}{|c|}{$\begin{array}{lllllllllllllllllllllll}\text { P1 } & \text { P2 } & \text { P3 } & \text { P4 } & \text { P5 } & \text { P6 } & \text { P7 } & \text { P8 } & \text { P9 } & \text { P10 } & \text { P11 } & \text { P12 } & \text { P13 } & \text { P14 } & \text { P15 } & \text { P16 } & \text { P17 } & \text { P18 } & \text { P19 } & \text { P20 }\end{array}$}} \\
\hline & & & & & & & & & & & & & & & & & & & & \\
\hline Nematomorpha & & & & $x$ & & & & $X$ & & & & & & & & & & & & \\
\hline \multicolumn{21}{|l|}{ Annelida } \\
\hline Hyrundinae & & & $x$ & & & & & $X$ & & $x$ & & & $x$ & & & $x$ & $X$ & & $X$ & \\
\hline Oligochaeta & $x$ & $x$ & $x$ & $x$ & $x$ & $x$ & $x$ & $x$ & $x$ & $x$ & $x$ & $x$ & $x$ & $x$ & $x$ & $x$ & $x$ & $x$ & $x$ & $x$ \\
\hline \multicolumn{21}{|l|}{ Mollusca } \\
\hline Bivalvia & & $X$ & & $x$ & $X$ & $x$ & & $X$ & & $x$ & $x$ & $x$ & $x$ & $X$ & $x$ & $x$ & $X$ & $x$ & $X$ & $X$ \\
\hline \multicolumn{21}{|l|}{ Gastropoda } \\
\hline Ampullariidae & & & & & & & & & & & & & $x$ & $X$ & & & & & & \\
\hline Planorbidae & & & $x$ & $x$ & & & & $X$ & & & & & $x$ & $X$ & $x$ & $x$ & $X$ & & & $X$ \\
\hline Lymnaeidae & & $x$ & & $x$ & & & & & & & & $x$ & & & $x$ & $x$ & $X$ & & & $x$ \\
\hline Thiaridae & & $X$ & $x$ & $x$ & & & & & & & & $x$ & & $X$ & $x$ & $x$ & $x$ & & & \\
\hline Pomaceae & & & & & & & & & & & & & & $x$ & & & & & & $x$ \\
\hline \multicolumn{21}{|l|}{ Arthropoda } \\
\hline \multicolumn{21}{|l|}{ Chelicerata } \\
\hline \multicolumn{21}{|l|}{ Arachnida } \\
\hline Hydracarina & $x$ & $x$ & $x$ & $x$ & $x$ & $x$ & $x$ & & $x$ & $x$ & $x$ & $x$ & & & & & & & & \\
\hline \multicolumn{21}{|l|}{ Crustacea } \\
\hline \multicolumn{21}{|l|}{ Malacostracoda } \\
\hline \multicolumn{21}{|l|}{ Decapoda } \\
\hline Paleomonidae & & $x$ & $x$ & $x$ & & & & & & & & $x$ & & $x$ & & $x$ & $x$ & & & \\
\hline \multicolumn{21}{|l|}{ Amphipoda } \\
\hline Hyalidae & & & $x$ & & & & & & & & $x$ & & $x$ & & & & & & & \\
\hline \multicolumn{21}{|l|}{ Branchiopoda } \\
\hline Cladocera & & & & & & & $x$ & & & & & & & & & & & & & \\
\hline Ostracoda & $x$ & & & $x$ & & $x$ & & $x$ & & & & & & $X$ & & $x$ & $X$ & & $X$ & $X$ \\
\hline
\end{tabular}


Tabela 2. Continuação.

Table 2. Continuation.

\begin{tabular}{|c|c|c|c|c|c|c|c|c|c|c|c|c|c|c|c|c|c|c|c|c|}
\hline Taxa & P1 & P2 & P3 & $\mathrm{P} 4$ & P5 & P6 & P7 & P8 & P9 & P10 & P11 & P12 & P13 & P14 & P15 & P16 & P17 & P18 & P19 & P20 \\
\hline Copepoda & & & & & & $X$ & $X$ & & & & & & & & & & & & & \\
\hline \multicolumn{21}{|l|}{ Atelocerata } \\
\hline \multicolumn{21}{|l|}{ Hexapoda } \\
\hline Collembola & & & $x$ & & & & & & & & & & & & $X$ & & & $x$ & & \\
\hline \multicolumn{21}{|l|}{ Insecta } \\
\hline \multicolumn{21}{|l|}{ Ephemeroptera } \\
\hline Leptophlebiidae & $x$ & $x$ & $X$ & $x$ & $X$ & $x$ & $X$ & $X$ & $x$ & $x$ & $x$ & $X$ & $x$ & $x$ & $X$ & & & & $X$ & $x$ \\
\hline Leptohyphiidae & $X$ & $x$ & $X$ & $X$ & $X$ & $X$ & $X$ & $X$ & $x$ & $X$ & $\mathrm{X}$ & $X$ & $X$ & $X$ & $X$ & $\mathrm{X}$ & $X$ & $X$ & $X$ & $X$ \\
\hline Baetidae & $X$ & $X$ & $X$ & $X$ & $X$ & $X$ & $X$ & $X$ & $X$ & $X$ & $X$ & $X$ & $X$ & $X$ & $X$ & $X$ & $X$ & $X$ & $X$ & $x$ \\
\hline Caenidae & & $x$ & & & & $x$ & & $X$ & $x$ & $x$ & & & & $x$ & $X$ & & & & $X$ & \\
\hline Oligoneuriidae & $x$ & $x$ & & & $x$ & $x$ & & & & & $x$ & & & & & & & & & \\
\hline Polymitarcyidae & & & & & & & & & $X$ & & & & & & & & & & & \\
\hline Euthyplociidae & & $X$ & & & & & $x$ & $X$ & $X$ & $X$ & & & & & & & & & & \\
\hline \multicolumn{21}{|l|}{ Odonata } \\
\hline \multicolumn{21}{|l|}{ Zygoptera } \\
\hline Coenagrionidae & $X$ & $X$ & $X$ & $x$ & $\mathrm{X}$ & & $\mathrm{X}$ & $X$ & $X$ & $X$ & $X$ & $x$ & $X$ & $X$ & $X$ & $X$ & $X$ & & $X$ & \\
\hline Calopterigidae & & & & $X$ & $X$ & $X$ & & & & $X$ & & & $X$ & & $X$ & & & & & \\
\hline \multicolumn{21}{|l|}{ Anisoptera } \\
\hline Libellulidae & $X$ & $x$ & $X$ & $x$ & $X$ & $X$ & $x$ & $X$ & $X$ & $X$ & $\mathrm{X}$ & $X$ & $X$ & $X$ & $X$ & $\mathrm{X}$ & $X$ & $X$ & $X$ & $X$ \\
\hline Gomphidae & $\mathrm{X}$ & $x$ & $X$ & $x$ & $X$ & $x$ & $x$ & $X$ & $x$ & $x$ & $x$ & $X$ & $\mathrm{X}$ & $x$ & $X$ & $\mathrm{X}$ & $X$ & & $X$ & $X$ \\
\hline Aeshnidae & & & & & & & & & & & & & $\mathrm{X}$ & $x$ & & & & & & \\
\hline \multicolumn{21}{|l|}{ Plecoptera } \\
\hline Perlidae & $x$ & $x$ & & $x$ & $x$ & $x$ & $x$ & & & $x$ & $x$ & $X$ & & & $x$ & & & & & \\
\hline Hemiptera & & & & & & & & & & & & & & & & & & & & \\
\hline Pleidae & & & $x$ & $x$ & $x$ & & $x$ & & & & & $X$ & $X$ & & & & & & & \\
\hline Naucoridae & $x$ & $x$ & $x$ & $x$ & $x$ & & $x$ & $X$ & & $x$ & $x$ & $X$ & $X$ & & $X$ & & & $x$ & & $x$ \\
\hline Notonectidae & & & & & & $x$ & & $X$ & & & & & & $x$ & & $x$ & $X$ & $X$ & & \\
\hline Gerridae & & & & & $X$ & $x$ & & $x$ & & $x$ & $x$ & $X$ & & & & & & & & \\
\hline Veliidae & & $x$ & & & $X$ & & & & & $x$ & & $x$ & $x$ & & & & & & & \\
\hline Mesoveliidae & & & & & & & & & $x$ & $x$ & & & & & & & & & & \\
\hline Hebridae & & & & & & $X$ & & $x$ & & & & & & & & & & & & \\
\hline Corixidae & & & & & & & & $x$ & & & & & & $x$ & & $X$ & $X$ & & & \\
\hline Belostomatidae & & & & & & & $x$ & $x$ & & & & & & & & & & & & \\
\hline Megaloptera & & & & & & & & & & & & & & & & & & & & \\
\hline Corydalidae & $x$ & $x$ & & $x$ & $x$ & & $x$ & & & $x$ & & & & & & & & & & \\
\hline Trich & & & & & & & & & & & & & & & & & & & & \\
\hline Hydropsychidae & $X$ & $x$ & $X$ & $x$ & $x$ & $X$ & $x$ & $x$ & $x$ & $x$ & $x$ & $x$ & $X$ & $X$ & $X$ & $X$ & $X$ & & $X$ & $x$ \\
\hline Glossosom & & & & $x$ & & & $x$ & & $x$ & & & & & & $X$ & & & & & \\
\hline Leptoceri & $x$ & $x$ & $x$ & $x$ & $x$ & $x$ & $x$ & & $x$ & $x$ & $x$ & $x$ & $X$ & $x$ & $x$ & $x$ & $X$ & & $X$ & $x$ \\
\hline Helico & $x$ & $x$ & $X$ & $x$ & & $x$ & $x$ & $x$ & & $X$ & $x$ & $X$ & & & $X$ & & & & & $x$ \\
\hline Philopota & $x$ & $x$ & & $x$ & & & & & & $x$ & $x$ & & $x$ & & $X$ & & & & & \\
\hline Odontoc & $x$ & & $x$ & $x$ & & & & & $x$ & & & & $X$ & & $x$ & & & & & \\
\hline Hydroptilidae & $X$ & & & $x$ & $x$ & & $x$ & $X$ & $x$ & $x$ & $x$ & $X$ & $X$ & $x$ & $X$ & & & & & \\
\hline Polycentropodidae & & & & $x$ & $x$ & $x$ & $x$ & $X$ & $X$ & & & $x$ & $X$ & $x$ & $x$ & $x$ & $X$ & & $x$ & \\
\hline Lepdoptera & & & & & & & & & & & & & & & & & & & & \\
\hline Pyralidae & & $x$ & $X$ & $x$ & $X$ & & $x$ & $x$ & $X$ & $X$ & $x$ & $X$ & $X$ & $X$ & $X$ & $\mathrm{X}$ & $X$ & & & \\
\hline Orthoptera & $X$ & & & & $X$ & & $x$ & & & $X$ & & & & & & & & & & \\
\hline Coleoptera & & & & & & & & & & & & & & & & & & & & \\
\hline Hydrophilidae & $X$ & $X$ & & & & & & $X$ & & & & & $X$ & & $X$ & $X$ & $X$ & & $X$ & \\
\hline Elmidae & $X$ & $x$ & $X$ & $x$ & $X$ & $X$ & $X$ & $x$ & $x$ & $x$ & $x$ & $X$ & $X$ & $x$ & $X$ & $X$ & $X$ & $x$ & $X$ & $x$ \\
\hline Psephenidae & $x$ & $x$ & & & & & $x$ & & & & & & & & & & & & & \\
\hline Curculionidae & & & & $x$ & & & & & & & & & & & & $x$ & $X$ & $x$ & & \\
\hline Lutrochidae & & & $x$ & & & & & & & & & & & & & & & & & \\
\hline Scirtidae & & & $x$ & & & & & & & & & & & & $X$ & & & & & \\
\hline Gyrinidae & & & & $x$ & & & & $x$ & & & & & & & & & & & & \\
\hline Noteridae & & & $X$ & & & & & & & & & & & & $X$ & & & & & \\
\hline Dytiscidae & & $x$ & & $x$ & & & $X$ & $X$ & & $x$ & $x$ & & $X$ & & $X$ & $X$ & $X$ & $X$ & & \\
\hline Dipt & & & & & & & & & & & & & & & & & & & & \\
\hline Chironomidae & $X$ & $x$ & $X$ & $X$ & $x$ & $x$ & $x$ & $X$ & $X$ & $x$ & $x$ & $x$ & $X$ & $x$ & $X$ & $X$ & $X$ & $x$ & $X$ & $x$ \\
\hline gonidae & $X$ & $x$ & $x$ & $x$ & $x$ & $\mathrm{X}$ & $\mathrm{X}$ & $\mathrm{X}$ & $x$ & $x$ & $x$ & $x$ & $X$ & $x$ & $\mathrm{X}$ & $X$ & $X$ & $x$ & $X$ & $X$ \\
\hline Simullidae & $x$ & $x$ & $x$ & $x$ & $X$ & & $x$ & $X$ & $x$ & $x$ & $x$ & & $X$ & & $X$ & $x$ & $X$ & & $X$ & $x$ \\
\hline
\end{tabular}


Tabela 2. Continuação.

Table 2. Continuation.

\begin{tabular}{|c|c|c|c|c|c|c|c|c|c|c|c|c|c|c|c|c|c|}
\hline Taxa & P1 & P2 & P3 & $\mathrm{P} 4$ & P5 & P6 & P7 & P8 PC & P10 & P11 & P12 & P13 P14 & P15 & P16 & P17 P18 & P19 & P20 \\
\hline Empididae & $x$ & $X$ & $X$ & $X$ & $x$ & $X$ & $x$ & $x$ & $x$ & $x$ & & $x$ & $X$ & & & & $x$ \\
\hline Tabanidae & $x$ & & & & & & $x$ & $x$ & & & $X$ & & & $x$ & $x$ & & \\
\hline Tipulidae & $x$ & $X$ & $x$ & $x$ & $x$ & & $x$ & $x$ & & $x$ & & & & & & $X$ & \\
\hline Stratiomyidae & & $X$ & & & & & & & & & & & & & & & \\
\hline Muscidae & & & & & & & & & & & & & & & & & $\mathrm{X}$ \\
\hline Culicidae & & & & & & & & $x$ & & & & & $X$ & & & & \\
\hline
\end{tabular}

